\title{
An Eigenpoint Based Multiscale Method for Validating Quantitative Remote Sensing Products
}

\author{
Shaohui Chen and Hongbo Su \\ Key Laboratory of Water Cycle and Related Land Surface Processes, Institute of Geographic Sciences and Natural Resources Research, \\ Chinese Academy of Sciences, Beijing 100101, China \\ Correspondence should be addressed to Shaohui Chen; chensh@igsnrr.ac.cn
}

Received 8 October 2013; Revised 29 January 2014; Accepted 7 February 2014; Published 30 April 2014

Academic Editor: Shouting Gao

Copyright (C) 2014 S. Chen and H. Su. This is an open access article distributed under the Creative Commons Attribution License, which permits unrestricted use, distribution, and reproduction in any medium, provided the original work is properly cited.

This letter first proposes the eigenpoint concept for quantitative remote sensing products (QRSPs) after discussing the eigenhomogeneity and eigenaccuracy for land surface variables. The eigenpoints are located according to the á trous wavelet planes of the QRSP. Based on these concepts, this letter proposes an eigenpoint based multiscale method for validating the QRSPs. The basic idea is that the QRSPs at coarse scales are validated by validating their eigenpoints using the QRSP at fine scale. The QRSP at fine scale is finally validated using observation data at the ground based eigenpoints at instrument scale. The ground based eigenpoints derived from the forecasted QRSP can be used as the observation positions when the satellites pass by the studied area. Experimental results demonstrate that the proposed method is manpower-and time-saving compared with the ideal scanning method and it is satisfying to perform simultaneous observation at these eigenpoints in terms of efficiency and accuracy.

\section{Introduction}

Compared with the traditional observation based on discrete ground points, the quantitative remote sensing products (QRSPs) retrieved from electromagnetic signals with certain spectral bands provide two-dimensional distribution information for land surface variables for studying hydrology, geography, disaster, and so forth [1]. Therefore, validating the accuracy and reliability of the QRSPs is of vital importance for assessing these applications.

At the beginning of remote sensing, researchers knew the importance of validating the QRSPs and paid many efforts to do it; however, it remains an unsolved problem [2]. Becker and li [3] addressed the relationship between temperature, emissivity, and observation scales. Sellers et al. [4] assessed the progress of the last decade before 1995 in the areas of modeling, satellite data algorithm development, and field experiments. Baret et al. [5] indicated that the validating system and framework of the QRSPs should be erected from the interrelationship among the systems on the ground, and the condition, criterion, and principle used to select the validating field should be formulated according to the validated QRSPs. Jiang et al. [6] pointed that the key of the remote sensing validation system is the synchronous data acquirement. Zhang et al. [2] proposed an operational "one test and two matches" validation method by introducing information fractal to the QRSP after pointing out the differences and similarities between the scale-free phenomena of the QRSP and the measurements of the coastline length (1dimensional) and the curved surface area (2-dimensional).

In recent years, the validation of one QRSP has been extended to the validation of the multi-QRSPs. Taking the MODIS of the EOS project as an example, the validation involves the surface albedo, ground temperature, bidirectional reflectance, NDVI, LAI, the primary productivity of vegetation, aerosol optical thickness, and so forth. Aerosol robotic network and twenty-four land surface key validating fields over the world were erected in order to validate the models and the retrieval algorithms [7-12].

In the Heihe Watershed Allied Telemetry Experimental Research, a multiscale observation experiment on evapotranspiration (ET) was performed over heterogeneous land surfaces. Multi-instruments were intensively deployed to constitute an observing matrix for the development and validation of the ET estimation models. The intensive observations and field campaigns were orderly undertaken in 
the Heihe watershed $[13,14]$. The terrestrial environmental observations provide long-term measurements for validating the ET retrieved from remote sensing data from the viewpoint of scale matching.

So far, the QRSPs are usually validated using ground based observation data that are gathered in the coordinated field campaign which combines spaceborne, airborne, and ground based observations together [15]. Ideally, during the short simultaneous measurement interval when the airborne or satellite overpasses, the validating data are taken by simultaneous measurement using imaging spectroradiometer on one platform of remote sensing vehicle, such as autoboat and balloon, in manner of scanning without gap and overlap $[2,16]$. Figure 1 shows the schematic sketch of scanning observation. In Figure 1, $a$ denotes the field of view which covers the swath $(L), V$ denotes flight direction, $\beta$ denotes the IFOV, $\theta$ is the angle between heights $H_{0}$ and $H_{\theta}$, and $a_{0}$ and $a_{\theta}$ denote the spatial resolutions. Because the spatial scales of the QRSPs are often far larger than those of the observation data, it requires too many times of observations to finish the scanning for obtaining the validating data. It is often difficult to realize the scanning observation over the heterogeneous surface because the interval when the airborne or satellite passes by the sample area is very short [5]. What is more, it would consume great finance and manpower to perform the scanning observation because the instantaneous field of view (IFOV) of measuring instrument is much smaller than that of the airborne or satellite [17]. Practically, seldom observation data are obtained using this method.

However, without these observation data, the relationship could not be connected between the QRSPs and their reliability. In this letter, a more practical method based on eigenpoint observation is presented to replace the scanning method by introducing the concepts of eigenhomogeneity and eigenaccuracy into the validation of the QRSPs, exemplified by ground temperature product. Using the proposed method, it can be quick and exact to gather the required data, and it can also be extended to get data of other types, such as albedo and leaf area index, used to validate the corresponding QRSPs.

\section{The Eigenpoint Based Multiscale Method}

To validate the QRSP, the most important concept is the true value. In general, the true values for the QRSPs are obtained by instrument measurement. It is well known that any instrument has its own measurement accuracy, which gives the uncertainty level in the measurement process [2]. By taking the temperature measurement of land surface as an example, assuming that the temperature of land surface is $25.97^{\circ} \mathrm{C}$, the measured value will be $25^{\circ} \mathrm{C}$ for the thermometer with the precision $1^{\circ} \mathrm{C}, 25.9^{\circ} \mathrm{C}$ for the thermometer with the precision $0.1^{\circ} \mathrm{C}$, and $25.97^{\circ} \mathrm{C}$ for the thermometer with the precision $0.01^{\circ} \mathrm{C}[18]$. Which kind of the three thermometers is the most appropriate for measuring the temperature of the land surface? In practical, the thermometer that can satisfy the validating requirement and has the coarsest precision is chosen.
In the practical calculation, retrieval, and application of the QRSP, if one precision is sufficient to characterize the basic properties of surface energy balance and mass exchange, we call it eigenaccuracy. The value obtained using the instrument with this eigenaccuracy is called relative true value. Eigenaccuracy is related to the observed variable of land surface and is determined by the specific case of practical studies.

Ideally, if land surface is homogenous, the irradiance emitted by each component of the land surface is identical. The observation data of the whole surface is equal to that of each subsurface. However, there is no ideal homogeneous surface in the real world. So-called homogenous surface can be only considered when all the subsurfaces have identical relative true values on the basis of eigenaccuracy. If the land surface is homogenous under certain eigenaccuracy, in this situation, there only needs to be one observation for obtaining the validating data. If the land surface is heterogeneous under certain eigenaccuracy, the observed area must coincide with the area of the land surface in manner of scanning measurement. In fact, the precision of one sensor is the threshold value of deciding the homogeneity. When the spatial variety of the measured variable is less than the threshold value, it cannot be detected by this sensor, and the measured variable over the corresponding land surface is considered as homogenous.

For convenience, in this letter, the measured data obtained using instruments are considered as one kind of the QRSP. In this letter, the spatial variety of the QRSP is abstracted using á trous wavelet transform (AWT). The AWT can preserve the translation invariance; that is, a translation of the original signal necessarily implies a translation of the corresponding wavelet coefficients.

In the AWT, the translation invariance property is preserved by replacing the downsampling operation with upsampling the filters by $2^{j}$ where $j$ expresses the $j$ th decomposition level, and the upsampled filters are obtained by inserting $2^{j}-1$ zeroes between any couple of consecutive coefficients in the absence of decimation, before processing the $j$ th level. Hence, the name "à trous" means "with holes."

The basic idea of the AWT is to decompose the signal having finite energy into one sequence of approximation and detail channels, each of them with a different degree of resolutions and frequencies, by filtering the original signal through a bank of equivalent filters, given by the convolution of recursively upsampled versions of the low-pass filter $h$ and the high-pass filter $g$ of the analysis bank $[19,20]$. The detail channels are called wavelet planes with the same size as the original signal.

The AWT [21] is an undecimated nonorthogonal multiresolution decomposition defined by a filter bank $\left\{h_{j}\right\}$ and $\left\{g_{j}=\delta_{j}-h_{j}\right\}$, and the Kronecker operator $\delta_{j}$ denotes an allpass filter. In the two-dimensional case, the filters become $\left\{h_{i} h_{j}\right\}$ and $\left\{\delta_{i} \delta_{j}-h_{i} h_{j}\right\}$, which means that the twodimensional detail signal is given by the difference between two successive approximations.

The AWT assumes that the original signal $c_{0}(k, l)$ is the convolution of the real continuous world $f(x, y)$ and a scaling 


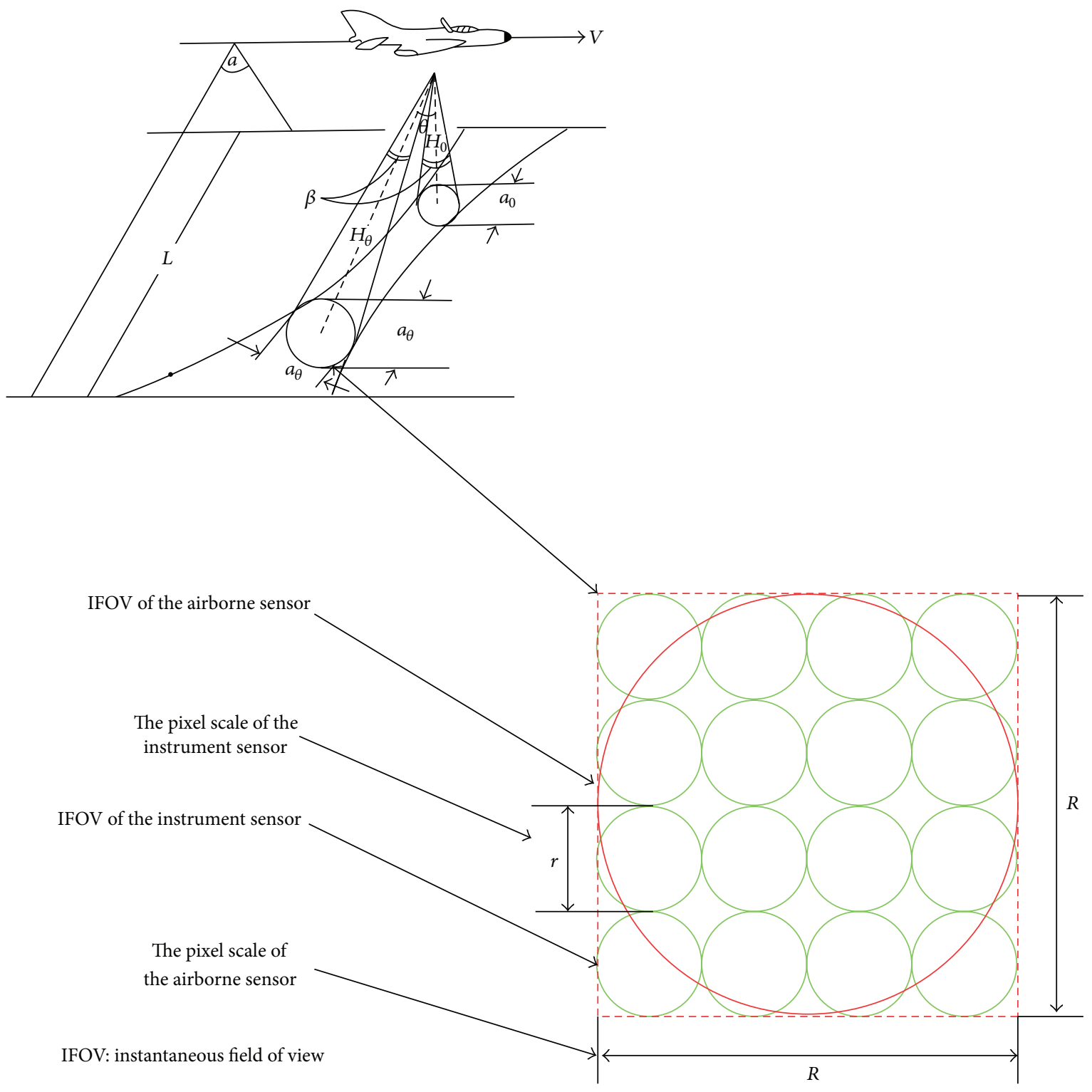

FIGURE 1: The schematic sketch of scanning observation.

function $\varphi(x, y)$ [22]. Approximate component $c_{j}(k, l)$ at scale $j$ is calculated by the following formula:

$$
c_{j}(k, l)=\frac{1}{4^{j}}\left\langle f(x, y), \varphi\left(\frac{x-k}{2^{j}}, \frac{y-l}{2^{j}}\right)\right\rangle .
$$

$\varphi$ satisfies the following dilation equation [23]:

$$
\frac{1}{2} \varphi\left(\frac{x}{2^{j}}, \frac{y}{2^{j}}\right)=\sum_{n} \sum_{m} h(n, m) \varphi\left(\frac{x}{2^{j-1}}-n, \frac{y}{2^{j-1}}-m\right) .
$$

$h(n, m)$ is the low-pass filter. According to (1) and (2), the approximate component $c_{j+1}(k, l)$ at scale $j+1$ is calculated by formula

$$
c_{j+1}(k, l)=\sum_{n} \sum_{m} h(n, m) c_{j}\left(k+2^{j} n, l+2^{j} m\right) .
$$

The wavelet plane $\omega_{j+1}(k, l)$ of scale $j+1$ is calculated by formula

$$
\omega_{j+1}(k, l)=c_{j}(k, l)-c_{j+1}(k, l) .
$$

The original signal can be reconstructed by adding the set of wavelet coefficients for all scales with the last approximation scale $c_{J}(k, l)[24]$ as

$$
c_{0}(k, l)=c_{J}(k, l)+\sum_{j=1}^{J} \omega_{j}(k, l) .
$$

In (5), $J$ denotes the number of decomposition levels. $\left\{\omega_{j}\right\}_{1 \leqslant j \leqslant J}$ denotes the wavelet coefficients. The AWT of an image is accomplished by a separated filtering following rows and columns, respectively. Specifically, a single wavelet plane 
is produced at each scale by subtraction of two successive approximations without decimation. Hence, wavelet and approximation planes have the same dimensions as the original image [25].

In this paper, $B_{3}$ spline wavelet filter is selected. The onedimensional low-pass filter is $h_{3}=[1 / 16,1 / 4,3 / 8,1 / 4,1 / 16]$ and the corresponding two-dimensional low-pass mask is

$$
\frac{1}{256}\left(\begin{array}{ccccc}
1 & 4 & 6 & 4 & 1 \\
4 & 16 & 24 & 16 & 4 \\
6 & 24 & 36 & 24 & 6 \\
4 & 16 & 24 & 16 & 4 \\
1 & 4 & 6 & 4 & 1
\end{array}\right) .
$$

In this letter, assuming that the $\mathrm{QRSP}^{t}$ denotes the product retrieved from remote data at temporal $t$. The AWT decomposition of the $\mathrm{QRSP}^{t}$ at the spatial location $(k, l)$ is as follows:

$$
\begin{aligned}
\operatorname{QRSP}(k, l) & =\operatorname{QRSP}_{J}(k, l)+\sum_{j=1}^{J} \mathrm{QRSP}_{J}(k, l), \\
S(k, l) & =\sum_{j=1}^{J} \operatorname{QRSP}_{J}(k, l) .
\end{aligned}
$$

The decomposition is completed when the following equation holds:

$$
\operatorname{Max}\left(\operatorname{QRSP}_{J}(k, l)\right)-\operatorname{Min}\left(\operatorname{QRSP}_{J}(k, l)\right) \leq P .
$$

In (9), $P$ is the given eigenhomogeneity. Then, determining the eigenpoints of the QRSP is transferred to determine the eigenpoints of $S$. In this letter, the spatial variety of $S$ is quantified using the standard deviation as

$$
\sigma_{S}=\left[\sum_{k} \sum_{l}(S(k, l)-\mu)^{2}\right]^{1 / 2},
$$

where $\sigma_{S}, S(k, l)$, and $\mu$, respectively denote the spatial variety, the $(k, l)$ value of $S$, and the mean value of $S$ :

$$
\begin{gathered}
\sigma_{y}=\sigma_{S} \leq P, \\
\sigma_{n}=\sigma_{S}>P,
\end{gathered}
$$

where $\sigma_{y}$ and $\sigma_{n}$ denote homogeneity and heterogeneity, respectively. According to (9), the more or the less homogenous the spatial distribution of the QRSP, the lower or the higher the standard deviation is, the less or the more the difference between $\sigma$ and $P$, and the higher or the lower the homogeneity is.

In order to get the observation data used to validate the QRSP, the challenging problem is that the IFOV of the instrument is usually much smaller than the IFOV of the sensor from which the QRSP is retrieved. One practical method is that the measured data obtained using the instruments are transformed to the spatial resolution of the QRSP by averaging; then the transformed data are used to validate the QRSP. This is called scale-by-scale or multiscale validation.
Assuming the spatial resolution ratio between the validated QRSP and the adopted instrument is $R$, we now determine the positions at which the validating data are measured. Based on (8)-(12), the following procedures constitute the multipoint observation based method (Figure 2).

(1) Find the function of the validated variable (such as temperature) varying with time. If there is no such function, construct it by analyzing the existing data which can be obtained using the instrument in manner of scanning.

(2) Use the function to forecast the image of the validated variable at the time when the satellite or the airborne passes by the ground. Decompose the forecasted image with the AWT. Consider the sum of the wavelet planes as the initial window, and push it into one stack.

(3) Compute the standard deviation of all windows in the stack using (10). If (11) holds for each window, then the process ends, and if (12) holds, go (4).

(4) Select the window with the largest standard deviation from the stack, and equally divide it into four parts; then insert them into the stack.

(5) Loop (3)-(4) until (11) holds for each window in the stack.

(6) The number and the positions of observation points are, respectively, obtained by the number and the centers of the windows in the stack. Here, observation points are called eigenpoints.

\section{Experimental Results and Analyses}

Figure 3 shows the schematic sketch of the simultaneous observation which combines the satellite, exemplified by Landsat thematic (TM) sensor with an IFOV of $30 \mathrm{~m}$, and the manual instrument on the ground, exemplified by thermal infrared radiometer with an IFOV of $0.5 \mathrm{~m}$ at a height of $1 \mathrm{~m}$. How and where do we collect the data required to validate the temperature product retrieved from the TM data using the thermal infrared radiometer on the ground? Because the interval during which the TM satellite passes by the ground is too short, it is difficult to perform scanning observation which requires many times of measurements using the thermal infrared radiometer. In this section, the proposed method is evaluated to substitute the scanning observation by selecting the ground observation points using existing thermal infrared temperature images (TITIs) during the simultaneous observation between the TM and thermal infrared radiometer sensors.

Assume we have $N$ TITIs $T=\left\{T_{1}, T_{2}, \ldots, T_{N}\right\}$, and the suffixes denote the imaging time. The simultaneous observation will happen at the next time $N+1$. Now, we want to determine the optimal points for the simultaneous observation using $T$. First we use $T$ to forecast the TITI at time $N+1\left(T_{N+1}\right)$ and then derive the observation points from $T_{N+1}$ using the proposed method. 


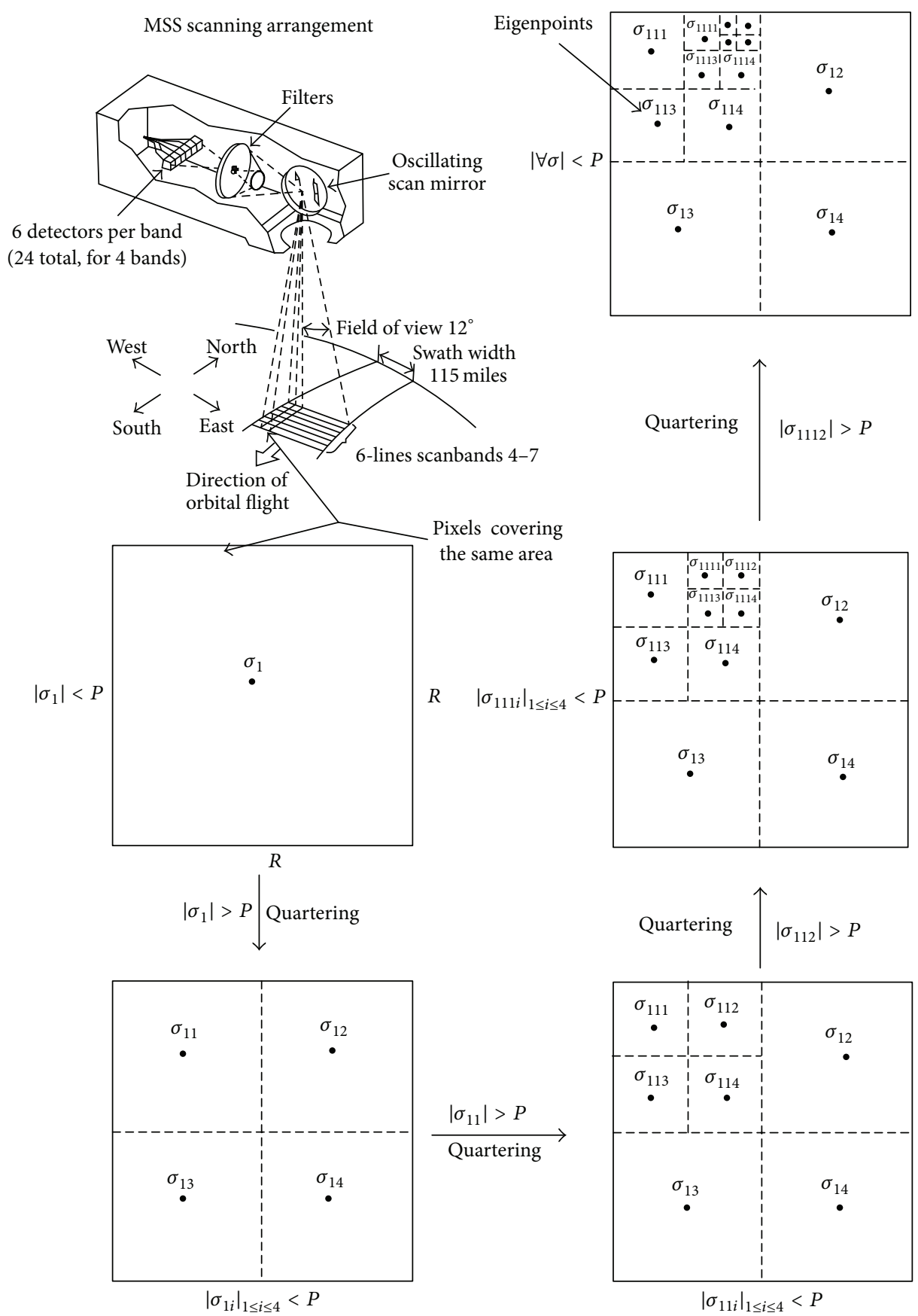

FIGURE 2: The four-level decomposition flowchart of the proposed method used to find eigenpoints.

Jackson et al. [26] considered that sun radiant intensity has a sin relationship with the sun radiance in a clear day. Furthermore, we consider that ground temperature varies with sun radiant intensity in a similar way as

$$
T_{t}=T_{\max } \sin \frac{t \pi}{\Omega} .
$$

In (13), $T_{\max }$ denotes the maximum thermal infrared temperature (TIT) of the studied surface when the sun is the only radiation source in a clear day. $T_{t}$ is the TITI of the studied surface at the time $t . \Omega$ denotes the hours of sunshine.
In order to obtain the sin function of each pixel of the studied surface from $T$, we construct the following equation:

$$
J_{(i, j)}=\sum_{n=1}^{N}\left(T_{n}(i, j)-T_{\max }(i, j) \sin \frac{n \pi}{\Omega}\right)^{2}
$$

In (14), $i, j$, and $(i, j)$, respectively, denote row, column, and location, $T_{n}(i, j)$ the TIT at $(i, j)$ at time $n$, and $T_{\max }(i, j)$ the maximum TIT at $(i, j)$ at the middle of sunshine hours. 


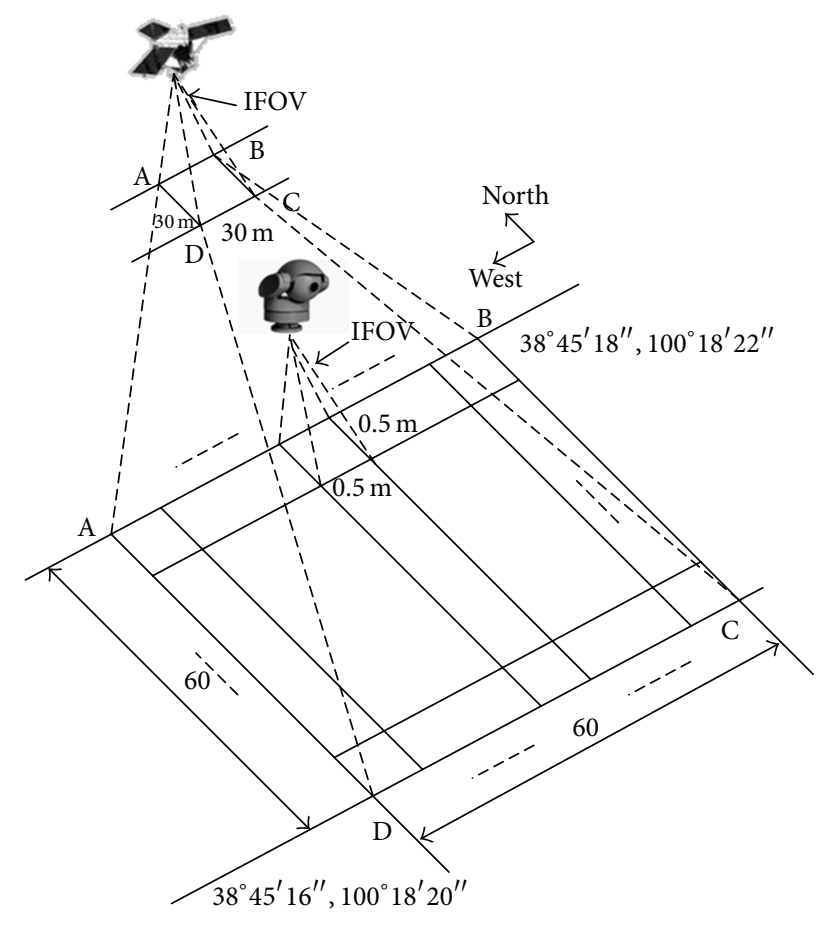

FIGURE 3: The sketch of the spaceborne and ground based simultaneous observations.

The solution of (14) can be gained using the least square method [27] as

$$
T_{\max }(i, j)=\frac{\sum_{n=1}^{N} T_{n}(i, j)}{\sum_{n=1}^{N} \sin (n \pi / \Omega)} .
$$

From (13) and (15), the TIT of $T_{N+1}$ at $(i, j)$ at time $N+1$ can be forecasted:

$$
T_{N+1}(i, j)=T_{\max }(i, j) \sin \frac{(N+1) \pi}{\Omega} .
$$

In the experiment, that is, the State Key Development Program for Basic Research of China with grant number 2007CB714401, nine TITIs are constructed using the observation data collected by the manual instrument on the ground. The temporal interval of these TITIs is 20 minutes from 7 to 10 a.m. on May 20, 2008, and the dimension of these TITIs is $60 \times 60$ at the spatial resolution $0.5 \mathrm{~m}$ corresponding to one pixel size of the TM data. The nine TITIs are used to obtain the sin function of thermal infrared temperature. The studied area covered by the these TITIs ranges approximately from $38^{\circ} 45^{\prime} 16^{\prime \prime}$ to $38^{\circ} 45^{\prime} 18^{\prime \prime}$ south latitude and $100^{\circ} 18^{\prime} 20^{\prime \prime}$ to $100^{\circ} 18^{\prime} 22^{\prime \prime}$ west longitude (Figure 3).

In this area, the sunshine hours are 13 from 6 a.m. to 7 p.m. The temperature attains the maximum value at 12.5 of the local time. This study area is located in the desert oasis of the middle reaches of the Heihe River Basin, Linze County, Gansu province. This area has a continental arid desert climate. Yearly averaged temperature is $7.6^{\circ} \mathrm{C}$, and the highest and lowest ones are, respectively, $-27.3^{\circ} \mathrm{C}$ in January and $39.1^{\circ} \mathrm{C}$ in July. The average canopy height is $5-6 \mathrm{~cm}$.

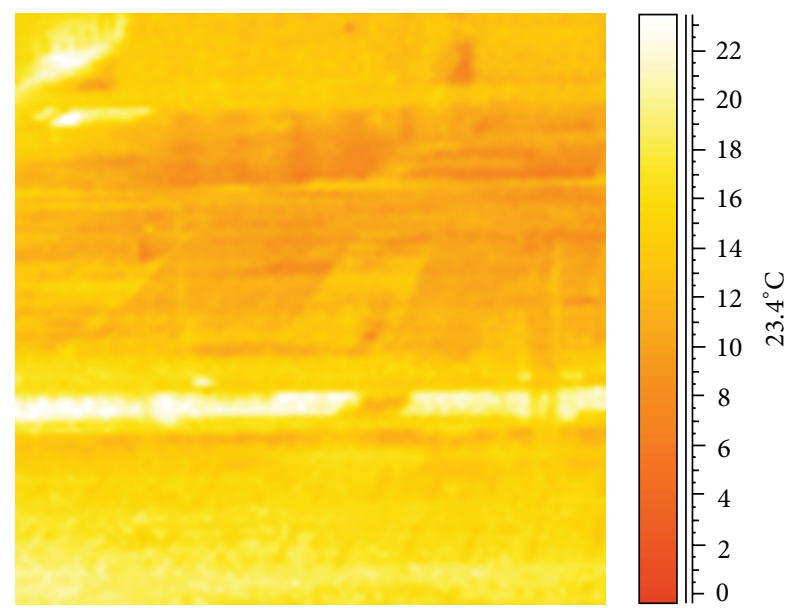

Figure 4: The TITI at 8 a.m. on May 20, 2008.

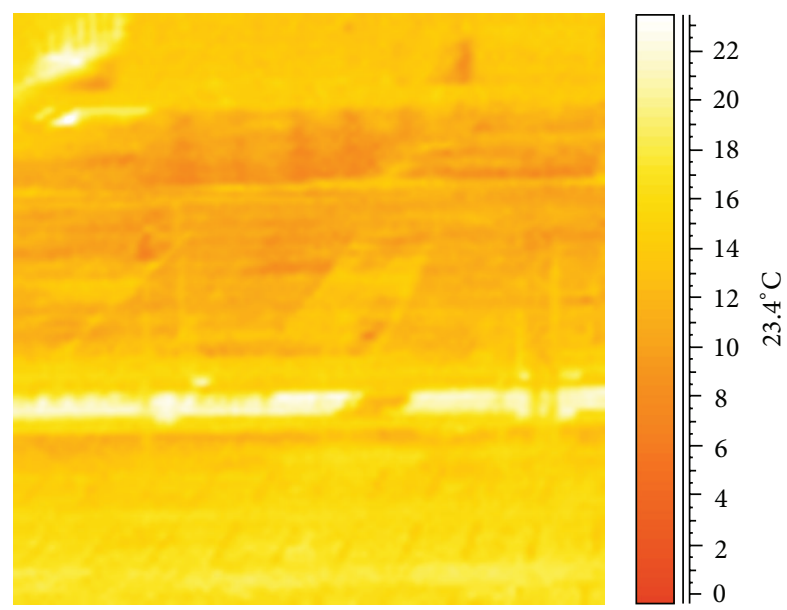

FIgURE 5: The TITI at 9 a.m.

The vegetation coverage is less than $10 \%$. Average annual rainfall is $117.1 \mathrm{~mm}$, up to $210.5 \mathrm{~mm}$, a minimum of only $82.9 \mathrm{~mm}$. Natural vegetation mainly includes bubble thorns, red sand, pearls, and other small shrubs. Soils are mainly gray-brown desert soil, sandy soil, and warping soil.

As two examples of the nine TITIs, Figures 4 and 5 show the two TITIs at 8 and 9 a.m. on May 20, 2008. According to the sin relationship, the TITI at the satellite overpassing time can be forecasted. Figures 6 and 7 show the two TITIs, respectively, obtained from the forecast and the scanning observation at 10.30 a.m. Then the proposed method is applied to the forecasted TITI to get the eigenpoints. Figures 8 and 9 show the eigenpoint distribution, respectively, obtained from Figures 6 and 7 using the proposed method. The obtained eigenpoints are used as the simultaneous observation points to collect observation values when the TM satellite passes. Figure 10 shows the eigenpoint distribution when the scanning is implemented. The validation work is conducted by using the observation values to validate the thermal infrared temperature retrieved from the TM data. Figures 4-10 are enlarged for better vision effect. 


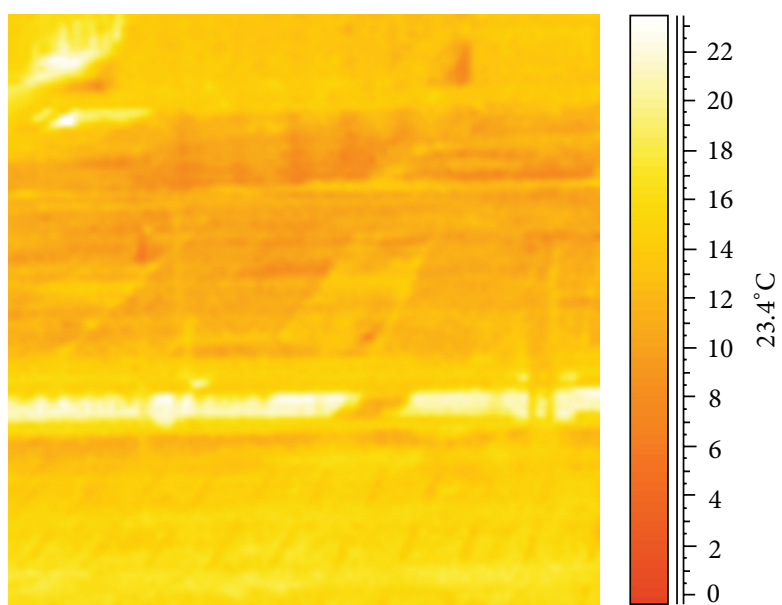

FIGURE 6: The forecasted TITI at 10.30 a.m.

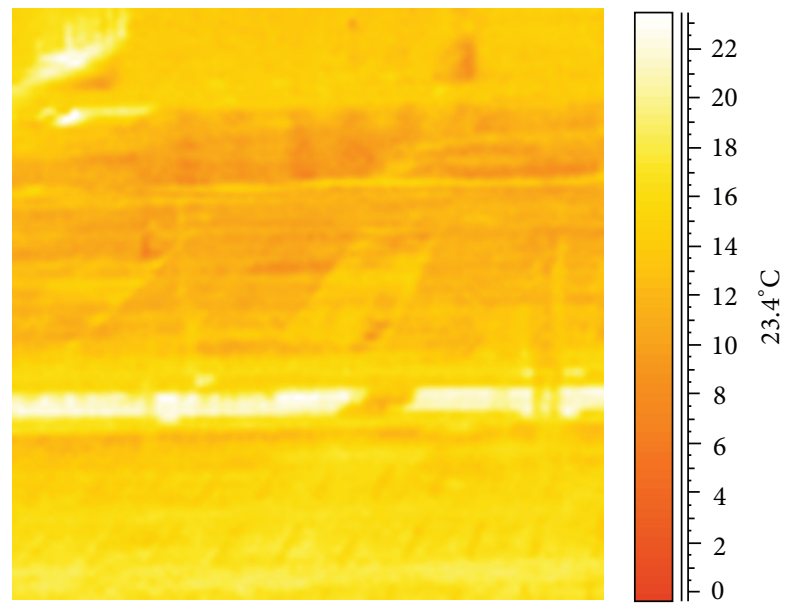

FIgURE 7: The constructed TITI at 10.30 a.m.

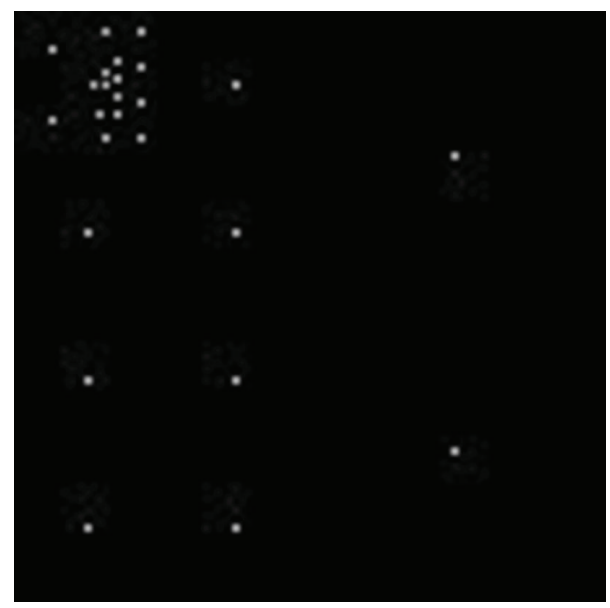

FIGURE 8: The eigenpoint distribution obtained from Figure 6 using the proposed method.

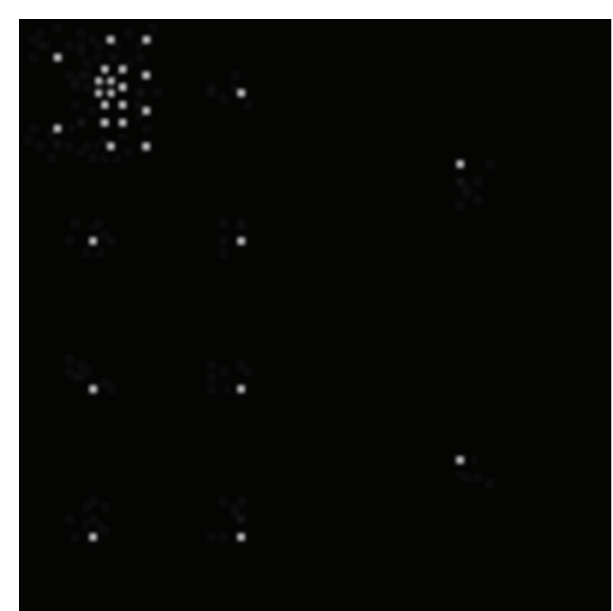

FIGURE 9: The eigenpoint distribution obtained from Figure 7 using the proposed method.

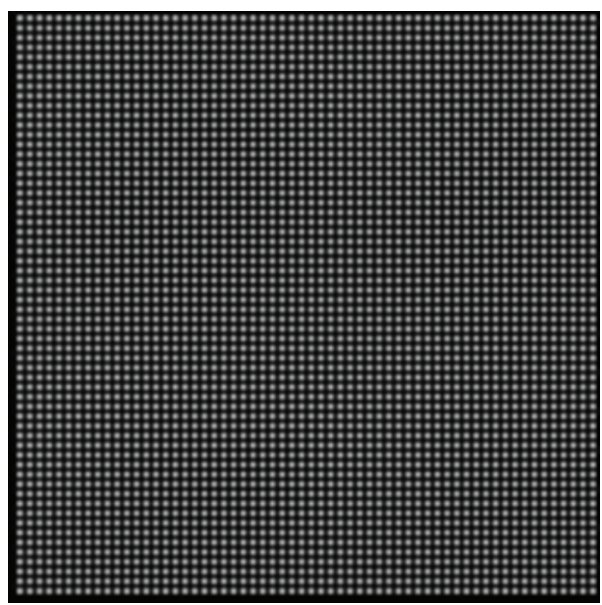

FIGURE 10: The observation point distribution of the scanning method.

In this experiment, the eigenhomogeneity $0.6^{\circ} \mathrm{C}$ is used, and $0.6^{\circ} \mathrm{C}$ is empirically determined because we considered that $0.6^{\circ} \mathrm{C}$ is sufficiently accurate for validating the temperature retrieved from the TM data when characterizing the surface energy balance and mass exchange. Six decomposition levels for the AWT are performed because too many decomposition levels did not do well to abstract the textural pattern of surface temperature while causing a lot of additional computation or too few decomposition levels would not fully abstract the variable texture of surface temperature. Table 1 shows the number of eigenpoints and the average TITs of these points, respectively.

The 2 nd and 3rd columns in Table 1 show the number of eigenpoints and the average TITs of these eigenpoints obtained from Figures 6 and 7 using the proposed method, respectively. The 4 th column in Table 1 shows the number and the average TIT of observation points when the scanning method is performed. For the purpose of comparison, the thermal infrared temperature retrieved from the TM data is added in the 5 th column in Table 1. 
TABLE 1: The number and average TITs of the obtained eigenpoints.

\begin{tabular}{lcccc}
\hline & \multicolumn{2}{c}{ The proposed method } & Scanning & \multirow{2}{*}{ TM } \\
& Figure 8 & Figure 9 & Figure 10 & \\
\hline Number & 25 & 28 & 3600 & 1 \\
Average TIT & $19.3^{\circ} \mathrm{C}$ & $19.6^{\circ} \mathrm{C}$ & $19.5^{\circ} \mathrm{C}$ & $19.2^{\circ} \mathrm{C}$ \\
\hline
\end{tabular}

It can be found from Table 1 that only 25 eigenpoints are selected from the forecasted TITI using the proposed method, which is less than the 28 points selected from the observation one and is much less than the 3600 points used in the scanning observation. Hence, if the measurements are only performed at the 25 points during the very short simultaneous time (usually fifteen minutes) when the satellite passes by the ground, it will take the least time and manpower to gather the required validating data.

From Figures 8 and 9, it also can be found that more eigenpoints are selected on the top left corner where the TITs of the bare and dry soil vary greatly with the sun radiant, while less eigenpoints are selected on the other parts where the TITs of the wet ground and vegetation vary slightly with the sun radiant. The second row in Table 1 shows the average TIT of these eigenpoints. It can be seen that though much less eigenpoints are used for the proposed method, the reliability of the validating data is still high by comparing $19.3^{\circ} \mathrm{C}$ and $19.5^{\circ} \mathrm{C}$. Compared with $19.5^{\circ} \mathrm{C}$, the difference between $19.3^{\circ} \mathrm{C}$ and $19.6^{\circ} \mathrm{C}$ is only $0.1^{\circ} \mathrm{C}$, and, in practice, this can be negligible. Hence, the performance of the proposed method is satisfying.

The eigenpoints displayed in Figure 8 are obtained from Figure 6 which is forecasted using (14), while the eigenpoints displayed in Figure 9 are obtained from Figure 7 constructed using the observation data of the scanning method. The difference of producing Figures 6 and 7 causes the difference in the eigenpoint location for Figures 8 and 9. The uncertainties come from three aspects: (1) the sin relationship of the thermal infrared temperature with time is not perfect and cannot exactly capture the relation between infrared temperature and time; (2) the points at which the scanning is performed are not the same for each scanning, which causes that the representation of the observation values from time to time may be different; (3) the time of the forecasted TITI and the constructed TITI is not exactly identical, which also produces difference.

Overall, the eigenpoints obtained can be used as observation positions with an accuracy level comparable to the scanning method. These results show that it is feasible to use the proposed method to forecast the eigenpoints in the satelliteground united field campaign in terms of efficiency and accuracy. It should be mentioned that the experiment is performed in the case of the time-dependent function is available. At present, the applicability of the eigenpoint method is restricted for the QRSPs that can be expressed as a function of time. Another limit of applying the proposed method is that more instruments than the scan method are required.

\section{Conclusions}

Validation plays a crucial role in quantitative remote sensing, which involves ground measurement, spaceborne, and airborne observations, model retrieval, QRSPs, and so on. It is difficult to finish the great number of measurements required in the ideal scanning observation without gap and overlap because the interval the satellite passes by the ground is very short. How to obtain the satisfying validating data with a few of measurements?

In this letter, an eigenpoint based multiscale observation method for validating the QRSPs is proposed in order to substitute the scanning method. This method originally relates the heterogeneity of the QRSP with the statistical indices of the á trous wavelet planes of the QRSP and defines the heterogeneity using the eigenhomogeneity and eigenaccuracy concepts. In this letter, eigenhomogeneity is defined using the standard deviation of pixel values. These two concepts are further introduced into determining the eigenpoints at which the obtained observation data used to validate the QRSPs are collected. When the difference between the mean value of the eigenpoint values of the QRSP and the mean value of the QRSP is less than the given eigenhomogeneity, these eigenpoints are the optimal observation positions, and the observation values obtained at these eigenpoints can be used as the validating data.

The scanning measurement is necessary to carry out the proposed method. For the places where it is hard to get the scanning observation, the historical data if available can be used as the substitution of the scanning observation. Fortunately, the underlying footprints where spaceborne, airborne, and ground coordinated observation happens are relatively homogeneous, and it is not often too difficult to perform the scanning observation.

In order to lessen the temporal variation in the eigenpoint location, it is better to take the scanning observation nearly before the coordinated observation. In order to lessen the spatial variation in the eigenpoint location, it is better to select the area where the underlying footprint is as homogeneous as possible as the battlefield where the coordinated observation is carried out.

Because there are various retrieved QRSPs, it is impossible to discuss all the QRSPs in one article. Hence, this letter takes the thermal infrared temperature as the paradigm. It is indeed difficult to construct the function of time for other land surface variables. The method proposed in this letter could not be applied to such kind of variable. However, it cannot constrain the application of the proposed method to other land surface variables that change with time, such as net radiant. In this instance, construct it by analyzing the existing data if there is no such function.

The significance of the proposed method comprises the following aspects.

(1) It can serve as a guideline for disposing the samples when there is a spaceborne, airborne, and ground coordinated observation.

(2) It can serve as an instruction in selecting the location for new planed field testing station. 
(3) It benefits to scaling [28] which is the next difficult problem for the QRSPs.

This letter makes forward a small step toward the validation of the QRSPs whose perfect realization still needs a long way to run. Without hesitation, the proposed method is inspiring, and it does provide a significant help for the validation of the QRSPs.

\section{Conflict of Interests}

The authors declare that there is no conflict of interests regarding the publication of this paper.

\section{Acknowledgments}

The authors thank the anonymous reviewers for their sincere suggestions which helped to improve the letter. This work is supported jointly by the Project of Natural Science Fund of China (41371348 and 41101329) and National Basic Research Program of China (2010CB950904 and 2010CB428403).

\section{References}

[1] R. H. Zhang, X. M. Sun, J. Y. Liu, H. Su, X. Tang, and Z. Zhu, "Determination of regional distribution of crop transpiration and soil water use efficiency using quantitative remote sensing data through inversion," Science in China D: Earth Sciences, vol. 46, no. 1, pp. 10-22, 2003.

[2] R. H. Zhang, J. Tian, Z. L. Li, H. B. Su, S. H. Chen, and X. Z. Tang, "Principles and methods for the validation of quantitative remote sensing products," Science China Earth Sciences, vol. 53, no. 5, pp. 741-751, 2010.

[3] F. Becker and Z. L. Li, "Surface temperature and emissivity at various scales: definition, measurement and related problems," Remote Sensing Reviews, vol. 12, no. 3-4, pp. 225-253, 1995.

[4] P. J. Sellers, B. W. Meeson, F. G. Hall et al., "Remote sensing of the land surface for studies of global change: models-algorithmsexperiments," Remote Sensing of Environment, vol. 51, no. 1, pp. 3-26, 1995.

[5] F. Baret, J. T. Morissette, R. A. Fernandes et al., "Evaluation of the representativeness of networks of sites for the global validation and intercomparison of land biophysical products: proposition of the CEOS-BELMANIP," IEEE Transactions on Geoscience and Remote Sensing, vol. 44, no. 7, pp. 1794-1802, 2006.

[6] X. G. Jiang, Z. L. Li, X. H. Xi, X. B. Li, and Z. Y. Li, "Basic frame of remote sensing validaion system," Arid Land Geography, vol. 31, no. 4, pp. 567-571, 2008.

[7] G. S. Jonathan, C. B. Schaaf, A. H. Strahler, F. Gao, and Y. Jin, "Validation of the MODIS bidirectional reflectance distribution function and albedo retrievals using combined observations from the aqua and terra platforms," IEEE Transactions on Geoscience and Remote Sensing, vol. 44, no. 6, pp. 1555-1564, 2006.

[8] S. Plummer, "On validation of the MODIS gross primary production product," IEEE Transactions on Geoscience and Remote Sensing, vol. 44, no. 7, pp. 1936-1938, 2006.

[9] T. M. Jeffery, F. Baret, J. L. Privette et al., "Validation of global moderate-resolution LAI products: a framework proposed within the CEOS land product validation subgroup," IEEE
Transactions on Geoscience and Remote Sensing, vol. 44, no. 7, pp. 1804-1817, 2006.

[10] M. Philippe, H. Eva, J. Gallego et al., "Validation of the global land cover 2000 map," IEEE Transactions on Geoscience and Remote Sensing, vol. 44, no. 7, pp. 1728-1739, 2006.

[11] I. A. Csiszar, J. T. Morisette, and L. Giglio, "Validation of active fire detection from moderate-resolution satellite sensors: the MODIS example in Northern Eurasia," IEEE Transactions on Geoscience and Remote Sensing, vol. 44, no. 7, pp. 1757-1764, 2006.

[12] D. Huang, W. Yang, B. Tan et al., "The importance of measurement errors for deriving accurate reference leaf area index maps for validation of moderate-resolution satellite LAI products," IEEE Transactions on Geoscience and Remote Sensing, vol. 44, no. 7, pp. 1866-1871, 2006.

[13] X. Li, S. Liu, M. Ma et al., "HiWATER: an integrated remote sensing experiment on hydrological and ecological processes in the Heihe River Basin," Advances in Earth Science, vol. 27, no. 5, pp. 481-498, 2012.

[14] H. Bogena, K. Schulz, and H. Vereecken, "Towards a network of observatories in terrestrial environmental research," Advances in Geosciences, vol. 9, pp. 109-114, 2006.

[15] K. Mallick, B. K. Bhattacharya, S. Chaurasia et al., "Evapotranspiration using MODIS data and limited ground observations over selected agroecosystems in India," International Journal of Remote Sensing, vol. 28, no. 10, pp. 2091-2110, 2007.

[16] S. H. Chen, H. B. Su, R. H. Zhang, J. Tian, and J. Xia, "Introducing object reflectance property and sensor spectral response into empirical mode decomposition based MODIS and TM image fusion," Canadian Journal of Remote Sensing, vol. 34, no. 6, pp. 585-595, 2008.

[17] E. P. Baltsavias, "A comparison between photogrammetry and laser scanning," ISPRS Journal of Photogrammetry and Remote Sensing, vol. 54, no. 2-3, pp. 83-94, 1999.

[18] R. H. Zhang, Z. L. Li, X. Z. Tang et al., "Study of emissivity scaling and relativity of homogeneity of surface temperature," International Journal of Remote Sensing, vol. 25, no. 1, pp. 245-259, 2004.

[19] L. Alparone, S. Baronti, A. Garzelli, and F. Nencini, "Landsat ETM+ and SAR image fusion based on generalized intensity modulation," IEEE Transactions on Geoscience and Remote Sensing, vol. 42, no. 12, pp. 2832-2839, 2004.

[20] M. Holdschneider, R. Kronland-Martinet, J. Morlet, and P. Tchamitchian, "A real time algorithm for the signal analysis with the help of the wavelet transform," in Wavelet, J. M. Combes, A. Grossman, and P. Tchamitchian, Eds., pp. 286-297, Springer, Berlin, Germany, 1989.

[21] F. Argenti and L. Alparone, "Speckle removal from SAR images in the undecimated wavelet domain," IEEE Transactions on Geoscience and Remote Sensing, vol. 40, no. 11, pp. 2363-2374, 2002.

[22] P. Dutilleux, "An implementation of the "algorithme à trous" to compute the wavelet transform," in Wavelets: Time-Frequency Methods and Phase Space, J. M. Combes, A. Grossman, and P. Tchamitchian, Eds., pp. 298-304, Springer, Berlin, Germany, 1989.

[23] G. Strang, "Wavelets and dilation equations: a brief introduction," SIAM Review, vol. 31, no. 4, pp. 614-627, 1989.

[24] Y. Chibani and A. Houacine, "The joint use of IHS transform and redundant wavelet decomposition for fusing multispectral and panchromatic images," International Journal of Remote Sensing, vol. 23, no. 18, pp. 3821-3833, 2002. 
[25] G. Pajares and J. M. de la Cruz, "A wavelet-based image fusion tutorial," Pattern Recognition, vol. 37, no. 9, pp. 1855-1872, 2004.

[26] R. D. Jackson, J. L. Hatfield, R. J. Reginato, S. B. Idso, and P. J. Pinter Jr., "Estimation of daily evapotranspiration from one time-of-day measurements," Agricultural Water Management, vol. 7, no. 1-3, pp. 351-362, 1983.

[27] M. L. Johnson and L. M. Faunt, "Parameter estimation by leastsquares methods," Methods in Enzymology, vol. 210, pp. 1-37, 1992.

[28] D. A. Quattrochi and N. S. Goel, "Spatial and temporal scaling of thermal infrared remote sensing data," Remote Sensing Reviews, vol. 12, no. 3-4, pp. 255-286, 1995. 

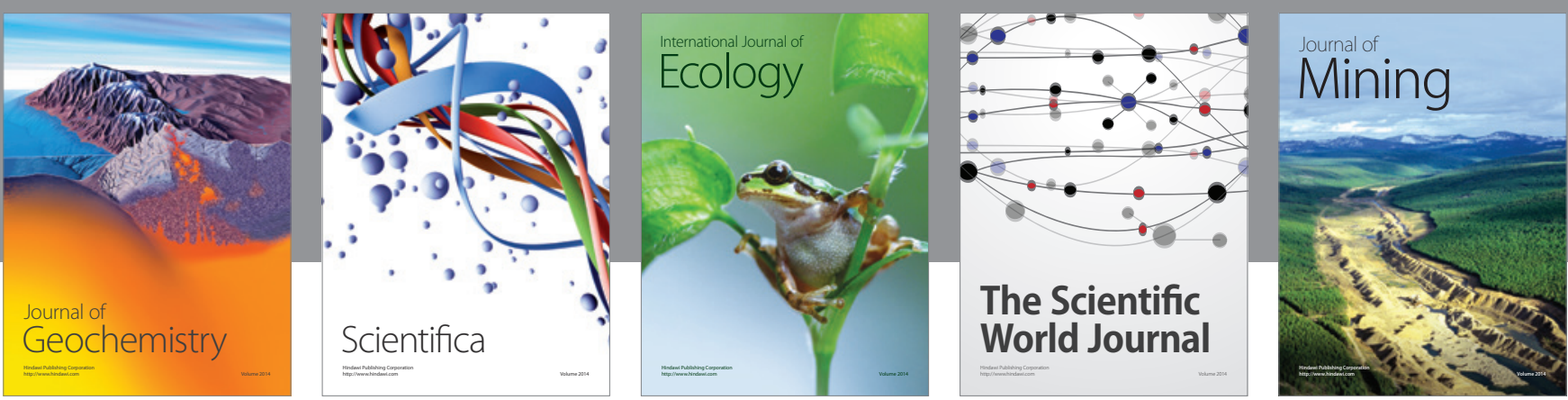

The Scientific World Journal
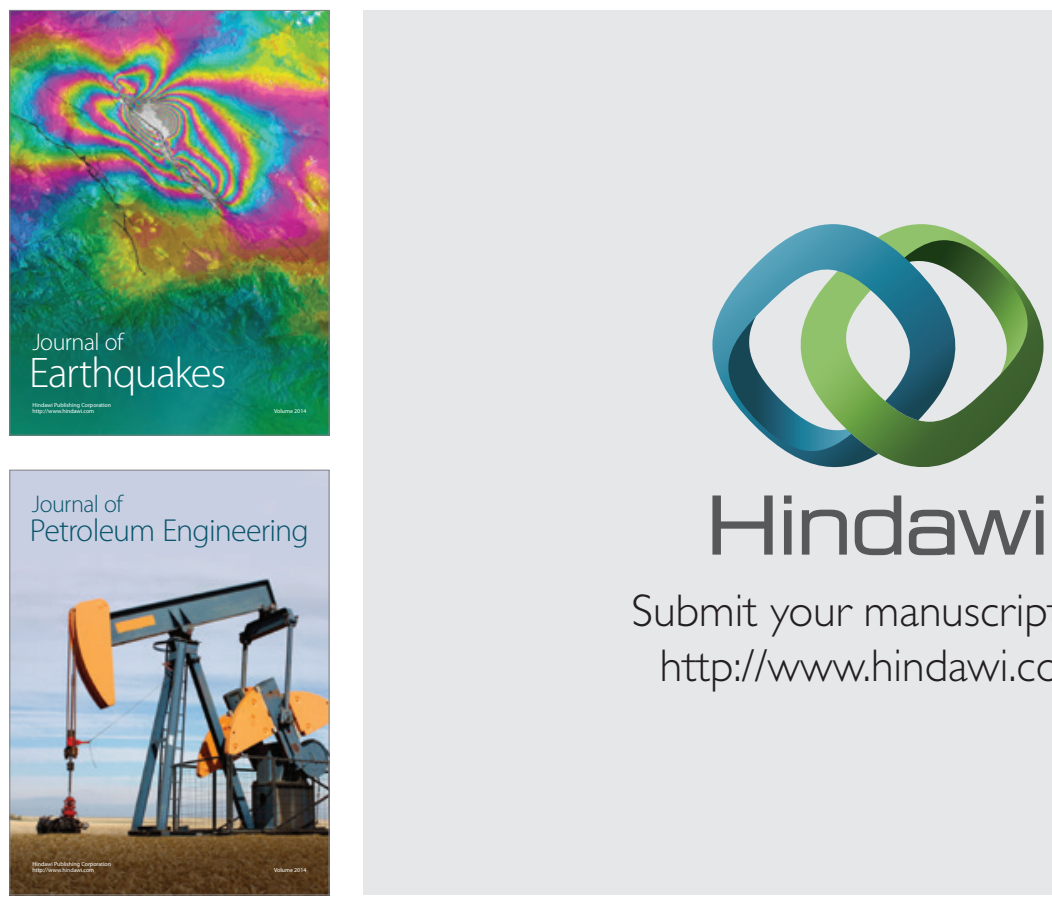

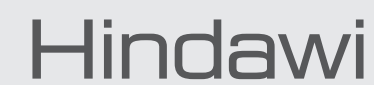

Submit your manuscripts at

http://www.hindawi.com
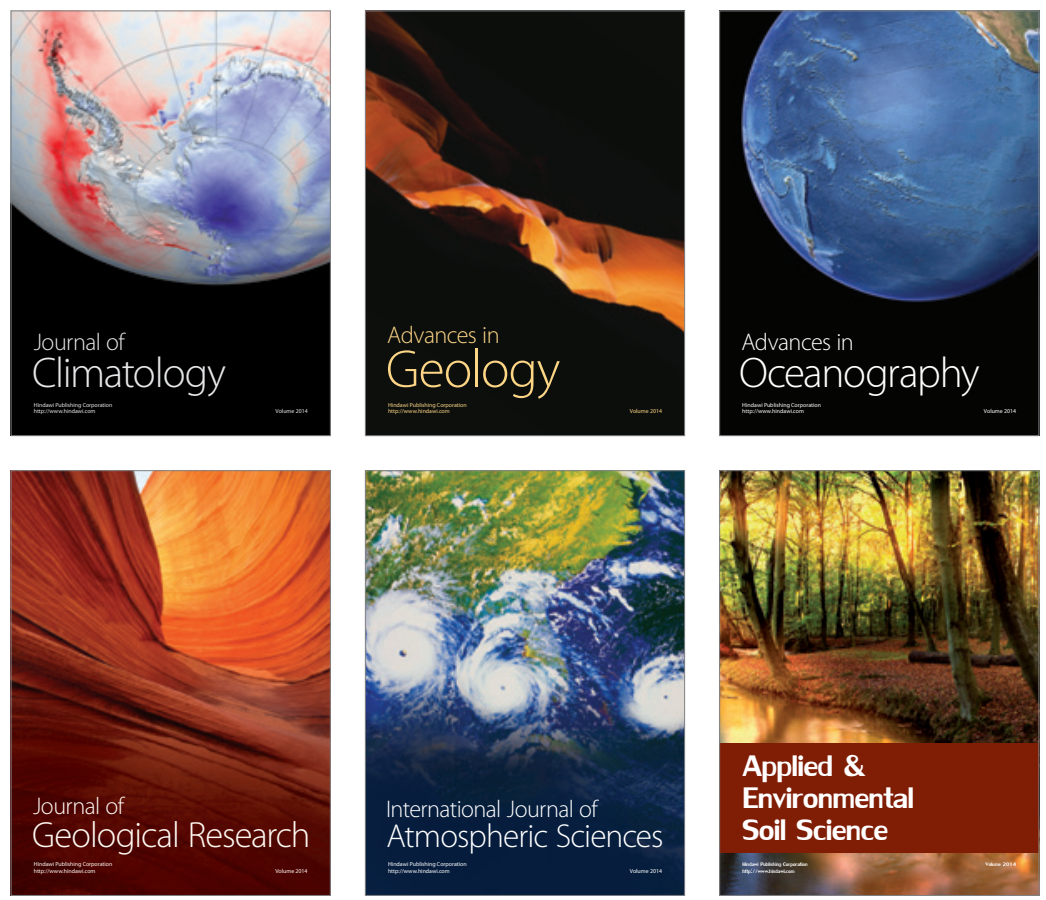
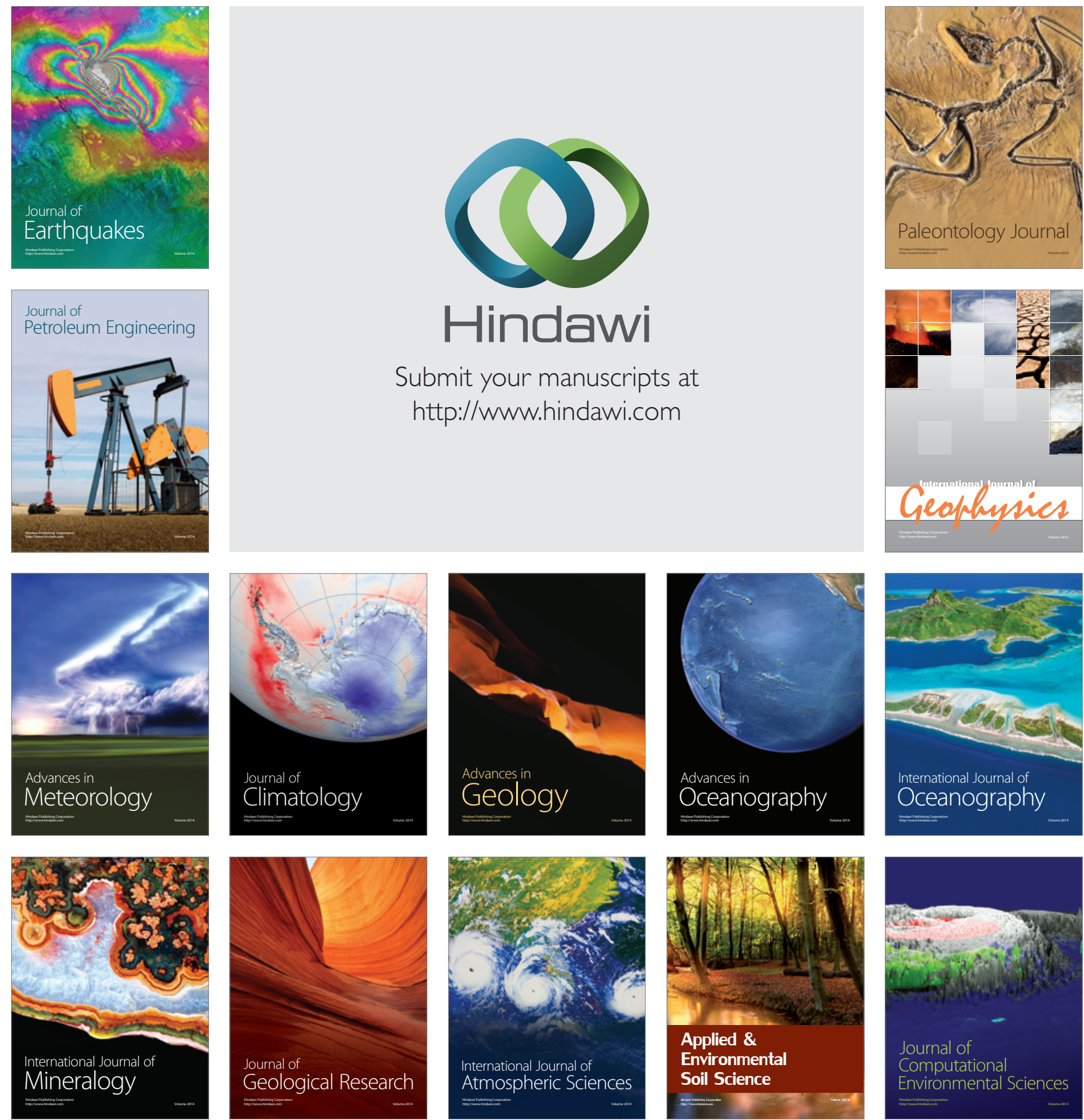